experience provides the substance for Jessen and Rosen's caution.

We are grateful to Professor Nimmo, of the Department of Physics, University of Otago, and to his staff for their valuable contribution to this study. We wish to thank Mr. Colin Booth and Miss Pamela Fraser, of the Department of Surgery, University of Otago, for technical help, and Miss Nancy Bowles for secretarial assistance.
REFERENCES

Borrie, J., and Lichter, I. (1964). N.Z. med. 7., 63, 31. Burchell, H. B. (1961). Circulation, 24, 161.

Jessen, C., and Rosen, J. (1963). Acta chir. scand., 125, 567.

Noordijk, J. A., Oey, F. T. I., and Tebra, W. (1961). Lancet, 1, 975. Swedberg, J., Johansson, B. W., Karnell, J., and Malm, A. (1963). Acta chir. scand., 125, 547.

Weinberg, D. I., Artley, J. L., Whalen, R. E., and McIntosh, H. D. (1962). Circulat. Res., 11, 1004

\title{
Classification of Chest Injuries as an Aid to Treatment
}

\author{
J. W. LLOYD,* F.F.A. R.C.S. ; A. CRAMPTON SMITH,* M.A., M.B., F.F.A. R.C.s. ; \\ B. T. O'CONNOR, $†$ M.S., M.CH.ORTH., F.R.C.S., F.R.A.C.S.
}

Brit. med. F., 1965, 1, 1518-1523

Intermittent positive-pressure respiration (I.P.P.R.) is now recognized as having an important part to play in the treatment of chest injuries (Barrett, 1960 ; Griffiths, 1960 ; Windsor and Dwyer, 1961 ; Garden and Mackenzie, 1963). Although treatment by this method gives good results, it should not be applied where a simpler method would suffice. I.P.P.R. is a severe strain on nursing staff, and because of its complexity carries a morbidity and even a mortality of its own.

We have found it of practical value to consider the treatment of patients with chest injuries in three phases. (1) The emergency treatment of a number of conditions common to many chest injuries. These conditions include pain, pulmonary compression, paradoxical respiration, lung contusion, shock, and coexisting injuries. (2) The classification of patients in mild, moderate, and severe groups by ventilatory capacity, and not by the anatomical extent of the injury; and the institution of the appropriate treatment. (3) The maintenance of treatment.

There are two factors which complicate the treatment of chest injuries and therefore the description of treatment. The first is that the conditions referred to under heading No. 1 above are interrelated. If pain is relieved the amount of paradoxical movement of the chest wall may decrease, and where a complicated form of treatment seemed inevitable a simpler one may suffice. The second is that although some patients can be placed in the correct group when first seen, in others the effect of treatment of the conditions mentioned under heading No. 1 must be observed before classification can be effected. Other patients, again, may move from one group to another during treatment.

The treatment of chest injuries will therefore be described by amplifying the headings mentioned above, although this means that the most important part of the communication-the classification of patients-appears late. The results of treatment of 121 patients, with illustrative case reports, are also presented.

\section{Conditions Common to Many Chest Injuries}

\section{Pain}

All except very minor chest injuries are painful. The patient in pain is handicapped in two ways: ineffective coughing leads to bronchial obstruction by mucus with absorption of air distal to the block and consequent atelectasis. His breathing is

* Nuffield Department of Anaesthetics, Radcliffe Infirmary, Oxford. t Accident Service, Radcliffe Infirmary, Oxford. shallow, and this (Ferris and Pollard, 1960) leads to a progressive fall in lung compliance owing to diffuse closure of alveoli throughout the lung substance. Pain prevents him from reversing this tendency by deep breathing.

Pain can be controlled by conventional analgesics with or without antagonists and by thoracic segmental extradural block. We have used morphine alone and in combination with amiphenazole or tetrahydroaminacrine. Both these antagonists allow a larger dose of morphine to be given without respiratory depression. We have obtained good results with a combination of morphine $15 \mathrm{mg}$. and tetrahydroaminacrine $20 \mathrm{mg}$.

Segmental thoracic extradural block (Simpson et al., 1961) involves the injection of local analgesic solution through a polyvinyl catheter placed in the thoracic extradural space. This provides complete relief from pain in the affected segments, and the restricted area of analgesia allows the use of small amounts of local anaesthetic solution, so that the fall in blood-pressure which accompanies an extensive extradural block is avoided. The relief from pain enables suitable patients to clear their lower airway by coughing and enables them to rest, and it improves morale. It will also, by allowing the patient to breathe more deeply and slowly, diminish the amount of paradoxical movement of a " floating" segment of chest wall. Continuous analgesia can be maintained by injections of lignocaine $1.5 \%$ through the catheter at intervals of 90 to 120 minutes, or by amethocaine (Pantocaine Plombe) at intervals of about three hours. An injection before physiotherapy allows coughing and deep breathing and will often be sufficient to prevent pulmonary complications. Conventional analgesics will also be necessary when the extradural analgesia is not continuous. A rigid aseptic technique for introducing the catheter is obligatory. Infection has not occurred in our patients in spite of the fact that catheters are sometimes left in place for long periods-in one case for 25 days.

\section{Pulmonary Compression}

Either pneumothorax or haemothorax, or both, will compress the lungs. If a leak from an air passage to the pleural space is valvular a tension pneumothorax may develop and affect not only ventilation but venous return to the right side of the heart. Tension pneumothorax may endanger life and require urgent treatment by inserting into the pleural cavity a drain connected to an underwater seal. The drain is usually placed in the second anterior intercostal space, and an emergency underwater seal drain may be made from a transfusion set. If there is evidence of blood in the pleural cavity a tube of larger bore 
should in addition be placed in a more dependent intercostal space and connected to another underwater seal. This second drain may be inserted if the air-drain indicates the presence of blood. A persistent air-leak from a drain suggests a ruptured bronchus and may indicate thoracotomy.

Herniation of abdominal contents through the diaphragm can also cause lung compression, and should be treated by endotracheal intubation and I.P.P.R. followed by reparative surgery to the diaphragm.

\section{Paradoxical Respiration}

This occurs most commonly when ribs have been fractured in two places and a segment of chest wall "floats." The floating segment moves paradoxically: during inspiration it is sucked in, while during expiration, and especially when the patient coughs, it balloons out. The lungs cannot be fully expanded and ventilation is impaired; in addition, during inspiration vitiated air (pendulum air) may pass from the lung on the injured side to the lung on the intact side, further decreasing total ventilation. Paradoxical movement may interfere with the "thoracic pump," decreasing venous return and reducing cardiac output. Paradoxical movement of a large segment or segments of chest wall may be fatal, but even the worst cases can be satisfactorily managed by reversing the normal intrathoracic pressure gradients by using I.P.P.R. as in anasthesia for thoracic surgery (Nosworthy, 1951). This form of treatment is not always necessary, and much can be done to relieve a moderate degree of paradoxical respiration by relieving pain. Pain causes rapid shallow respiration, and this, by increasing the pressure gradients during respiration, tends to make paradoxical respiration worse. We have treated cases of moderate paradoxical movement of a "floating" segment of chest wall by extradural injection. After pain relief the patient breathes less rapidly and more deeply, his colour improves, and his ventilation, as judged clinically and by estimation of oxygen and carbon dioxide tensions in blood, approximates to normal.

\section{Lung Contusion}

This implies the extravasation of blood into alveoli and bronchi as a result of injury. Diffusion is interfered with, ventilation/perfusion ratios are upset, large right-to-left shunts can occur, and ventilation may be greatly impaired.

\section{Shock}

Patients with chest injuries may need large amounts of blood for resuscitation. Operative intervention, unless to control haemorrhage or repair a ruptured viscus, should be delayed until the blood volume has been restored to normal. Tracheotomy can safely be postponed if an endotracheal tube has been inserted.

\section{Coexisting Disease or Injury}

Chest injuries seldom occur alone. Head injuries of varying severity coexist in about $80 \%$ of the present series, intraabdominal injuries in $4 \%$, and fractures of long bones in $26 \%$.

Head Injury.-A coexisting head injury may modify the treatment of chest injuries. In an unconscious patient the tongue may obstruct the upper airway or pharyngeal contents may be inhaled. If unconsciousness is likely to be prolonged the insertion of a cuffed rubber tracheotomy tube may be indicated even though unnecessary for the treatment of the chest injury. In considering tracheotomy it must be remembered that a head injury will be made worse by any degree of anoxia due to an inadequately treated chest injury.
Intra-abdominal Injuries.-These injuries must be treated urgently, and usually by laparotomy. The additional restriction of coughing and breathing after operation, especially in patients with pre-existing chest disease, may indicate treatment by tracheotomy.

Cardiac Injuries.-The heart seems to escape injury remarkably often, but if bleeding into the pericardium occurs it must be treated by aspiration or by thoracotomy. Haemopericardium is suspected when the patient's condition deteriorates rapidly ir. the presence of a raised venous pressure and of distant heart sounds.

Fractures of Long Bones.-It may be difficult to change the position of a patient who has long-bone fractures immobilized by splints, and this may make a coincidental chest injury worse. It is probable that all long-bone fractures are accompanied by a greater or lesser degree of fat embolism, and if this is severe the associated pulmonary oedema may best be treated by tracheotomy and I.P.P.R. (Denman et al., 1964).

\section{Traumatic Thoracoplasty}

In some patients with fairly severe chest injuries who, however, can still ventilate satisfactorily when breathing spontaneously the chest wall begins to collapse after two to three days as a result of the action of atmospheric pressure on fractured ribs. This condition is sometimes called "traumatic thoracoplasty." Overriding of the fractured ribs is obvious on serial radiographs before collapse of the chest wall is clinically apparent. Traumatic thoracoplasty can be reversed by the early use of I.P.P.R., but if the condition is untreated considerable deformity and loss of lung function may follow.

\section{Classification}

When all the above priorities have been treated as energetically as possible the patient is classified as having a mild, moderate, or severe chest injury, so that appropriate further treatment can be given. His classification depends not on the anatomical extent of his injury but on his ability to ventilate satisfactorily, and this in turn depends on his ability to breathe and to clear his airway by coughing. The efficiency of the cough must be judged clinically, but in these patients it is very difficult to assess ventilation without measurements of respiratory minutevolume and of the $\mathrm{pH}, \mathrm{PCO}_{2}$, and $\mathrm{Po}_{2}$ of arterial blood.

Mild Chest Injury.--Some young patients with one or two fractured ribs can breathe and cough adequately and have no ventilatory impairment. Other patients are prevented by pain from breathing easily and from coughing. The only treatment they require is adequate relief from pain.

Moderate Chest Injury.-Patients in this group are those who in spite of pain relief cannot breathe or cough adequately. If the patient cannot cough for any of various reasonsparadoxical movement of a "floating segment," debility, reflex depression from a head injury-retention of bronchial secretions soon leads to atelectasis and underventilation, with consequent disturbance of $p \mathrm{H}, \mathrm{PcO}_{2}$, or $\mathrm{PO}_{2}$ of arterial blood. The patient in this group should be treated by tracheotomy, which will provide a route for the aspiration of secretions. It may also slightly reduce anatomical dead space.

Severe Chest Injuries.-The patients in this group are those who in spite of pain relief and tracheotomy still cannot ventilate properly, probably because of a major degree of paradoxical movement of a part of the chest wall or of lung contusion. Measurements of $\mathrm{pH}, \mathrm{PCO}_{2}$, and $\mathrm{PO}_{2}$ of arterial blood will confirm the inadequacy of ventilation. The treatment of these patients is by I.P.P.R. through the existing tracheotomy. After a few days the onset of traumatic thoracoplasty may also be an indication for I.P.P.R. 


\section{Results}

From January 1960 to January 1965121 patients with serious chest injuries have been treated in Oxford in this way, and all were seen by one or more of us. Thirty-three have required I.P.P.R. The results are shown in the Table. The mortality calculated on the final assessment was nil in group 1, two in group 2 , nine in group 3 -a total of 11 patients $(9.09 \%)$.

\begin{tabular}{c|c|c|c|c|c}
\multicolumn{7}{c}{ Results in 121 Cases } \\
\hline \multirow{2}{*}{ Initial } & \multicolumn{2}{|c|}{ Deteriorating } & $\begin{array}{c}\text { Final } \\
\text { Assessment }\end{array}$ & Mortality \\
\cline { 2 - 6 } & & To Group 2 & To Group 3 & To & 0 \\
Group 1 & 78 & 5 & 3 & 70 & 2 \\
Goup 2 & 28 & - & - & 33 & 9 \\
\hline Total & 15 & - & 15 & 18 & $11(9 \cdot 09 \%)$ \\
\hline
\end{tabular}

In the same period about another 400 cases of mild chest injury were admitted to the United Oxford Hospitals. Two patients with multiple injuries, including chest injuries, who died within a few minutes of admission have been excluded.

\section{Maintenance}

Group 1. Mild Chest Injuries.-The patient in this group may need to have analgesic administered or to have "top-up" doses of local analgesic injected through his extradural catheter. He must be kept under careful observation, because in spite of pain relief his ventilatory function may deteriorate and he may require more complicated forms of treatment. Careful measurements of pulse, blood-pressure, and respiratory rate, together with measurements of vital capacity or peak expiratory flow rate, will give early indication of deterioration. Adequate hydration will prevent bronchial secretions from becoming viscous, and physiotherapy two or three times a day will help the patient to expectorate. Daily radiographs of the chest will also be helpful to establish deterioration or improvement.

Group 2. Moderate Chest Injuries.-The management of a patient with a chest injury treated by tracheotomy is exacting. He requires all the measures applicable to the previous group, and, in addition, his tracheotomy poses special problems. As a rule we use a cuffed tracheotomy tube to avoid the risk of chest complications due to inhalation of pharyngeal contents. This means that all the patient's inspired air bypasses the upper air passages and is neither warmed nor humidified. Only careful attention to artificial humidification will avoid "crusting" of bronchial secretions which may block the tracheotomy tube. This may be done by a hot-water humidifier (Spalding, 1956), by nebulizers, or by the installation of saline into the trachea (Marshall, 1964). The greater severity of the chest injury in group 2 means that an even closer watch must be kept to ensure early detection of deterioration of ventilatory function. Fortunately, the presence of a cuffed tracheotomy tube makes measurement of respiratory minute-volume, vital capacity, and $\mathrm{PCO}_{2}$ by rebreathing method (Campbell and Howell, 1962) much easier.

Group 3. Severe Chest Injuries.-Patients receiving I.P.P.R. are managed along the lines used in the Respiration Unit in Oxford (Spalding and Smith, 1963). Patients with chest injuries are relatively easy to manage on respirators, provided their coincidental injuries are not too severe and they are not depressed by drugs or relaxants. Pulmonary infection usually improves, probably because the patient can make some attempt at coughing and can assist the nurse who is aspirating secretions. Care must nevertheless be taken to avoid cross-infection during aspiration. The accurate observation of expired respiratory minute-volume, tracheal pressure, pulse, bloodpressure, and temperature is necessary to detect any change in the patient's condition, and these procedures should be supplemented by measurements of blood $\mathrm{Po}_{2}, \mathrm{PCO}_{2}$, and $p \mathrm{H}$.
Patients who require I.P.P.R. for reasons other than trauma usually absorb well from the alimentary tract and can be fed by means of naso-gastric tubes from the onset. Severely injured patients do not usually absorb from the stomach, and require intravenous fluids for a few days. Feeding by naso-gastric tubes is begun as soon as there is evidence of absorption. Frequent determinations of blood electrolyte content must be made and an accurate chart of the intake and output of fluid must be kept. The specific gravity of each specimen of urine should be measured. Acute renal tubular necrosis may occur in seriously injured patients, due either to injury to the kidney or secondary to hypotension or anoxic anoxia.

Fractured ribs stabilize after 10 to 14 days and the patient is able to breathe without assistance for short periods. When the patient has been "weaned" from the respirator and swallowing is satisfactory the cuffed tracheotomy tube is replaced by a silver one. Inspiration now takes place partly through the natural upper airway and partly around the silver tracheotomy tube. Humidification of the inspired gases is no longer required and the patient can speak.

\section{Case Reports}

Case 1.-A 62-year-old man with chronic bronchitis was admitted with fractures of the upper six ribs on the right side and the lower three on the left. There was no paradoxical movement and the only factor limiting ventilation was pain. An extradural catheter was inserted at T 5 level and $9 \mathrm{ml}$. of $1.5 \%$ lignocaine was injected. Analgesia was excellent, but the extent of the nerve-block required to produce relief of pain lowered the blood-pressure to $60 \mathrm{~mm}$. $\mathrm{Hg}$ systolic, and he then complained of dizziness. This method of treatment was therefore abandoned in favour of conventional analgesics, and a tracheotomy became necessary the next day in order to aspirate secretions that he could not cough up because of inadequate pain relief. Five days later retained secretions became infected, sputum was copious and purulent, and ventilation was inadequate. I.P.P.R. was begun, and, after some difficulty in weaning, was discontinued seven days later. The patient then made an uneventful recovery.

Case 2.-A 50-year-old woman, also suffering from chronic bronchitis, was admitted with a mild head injury and with fractures of the second to sixth ribs on the left side with paradoxical movement of the chest wall. There were also fractures of the second and third ribs on the right side. An extradural catheter was inserted at $\mathrm{T} 5$ level and $5 \mathrm{ml}$. of $1.5 \%$ lignocaine gave complete relief from pain. This had the effect of diminishing the degree of paradoxical respiration, her colour improved, and she was able to breathe quietly at rest. Oxygen saturation of arterial blood on admission was $87 \%$, and the $\mathrm{PCO}_{2}$ was $48 \mathrm{~mm}$. $\mathrm{Hg}$. After the injection of analgesic solution into the extradural space the oxygen saturation rose to $90 \%$, and her $\mathrm{PCO}_{2}$ fell to $42 \mathrm{~mm}$. $\mathrm{Hg}$. Three days after admission she developed a chest infection and was unable to clear her lower airways by coughing. A tracheotomy was performed, and this, together with antibiotics, controlled the infection. Her tracheotomy was closed 14 days after admission.

Case 3.-A 43-year-old man was admitted from another hospital after a car accident. On admission he had fractures of the seventh, eighth, ninth, and tenth ribs on the right side with paradoxical movement and fractures of the sixth and seventh ribs on the left. There was a complete right pneumothorax and a small left pneumothorax, and also bilateral lung contusion. He also had comminuted closed fractures of the lower end of his left radius and ulna. Bilateral intercostal drains with underwater seals were introduced through the second intercostal spaces, and the lungs were re-expanded. After the infusion of 8 pints (4.5 litres) of blood his blood-pressure was still only $90 / 50 \mathrm{~mm}$. $\mathrm{Hg}$, and signs indicated an abdominal injury. At laparotomy a ruptured kidney was removed and a tracheotomy was performed at the end of the operation because it was felt that the chest injury and upper abdominal wound together would impair ventilation. Next day, when breathing air, his respiratory rate was $45 / \mathrm{min}$, and his inspired minute-volume $18 \mathrm{l}$./ min., his oxygen saturation was $82 \%$, and the $\mathrm{PCO}_{2} 41 \mathrm{~mm}$. $\mathrm{Hg}$. When breathing oxygen the $\mathrm{Po}_{2}$ was $113 \mathrm{~mm}$. $\mathrm{Hg}$. During the day it became clear that his high respiratory rate was tiring him, and I.P.P.R. was begun with a respiratory minute- 
volume of $18 \mathrm{l} . / \mathrm{min}$. and a frequency of $201 . / \mathrm{min}$. He adapted to the change of frequency without difficulty, and on the following day his $\mathrm{PCO}_{2}$ was $32 \mathrm{~mm}$. $\mathrm{Hg}$ and his $\mathrm{HbO}_{2} \% 86$. Two days later his $\mathrm{PCO}_{2}$ was $26 \mathrm{~mm}$. $\mathrm{Hg}$, and his $\mathrm{HbO}_{2} \% 94$. He was "weaned" from his respirator over the next two or three days, and his tracheotomy tube was removed 10 days after admission.

Case 4.-A 50-year-old man was admitted with concussion, a fractured left clavicle, and a fracture of the third rib on the right side. Because of the possible effect of conventional depressant analgesics on the head injury an extradural catheter was inserted at T 4 and the pain controlled by injection of $1.5 \%$ lignocaine. A radiograph on the following day showed the fourth to ninth ribs inclusive on the right side to be fractured in two places, and a day later there was overriding of the ribs (Fig. 1). I.P.P.R. was applied through id cuffed tracheotomy tube and the overriding was corrected (Fig. 2). He was disconnected from the respirator at intervals to allow him to clear his airways by coughing, and this was painless because of the extradural analgesia. I.P.P.R. was discontinued after eight days and the cuffed rubber tracheotomy tube exchanged for a silver one. The lungs remained fully expanded and the rib contour normal. The tracheotomy was closed on the 14th day after injury.

Case 5.-A 75-year-old man had six fractured ribs treated by pain relief with extradural analgesia. His ventilation remained adequate and he cleared his lower airways by coughing. Although

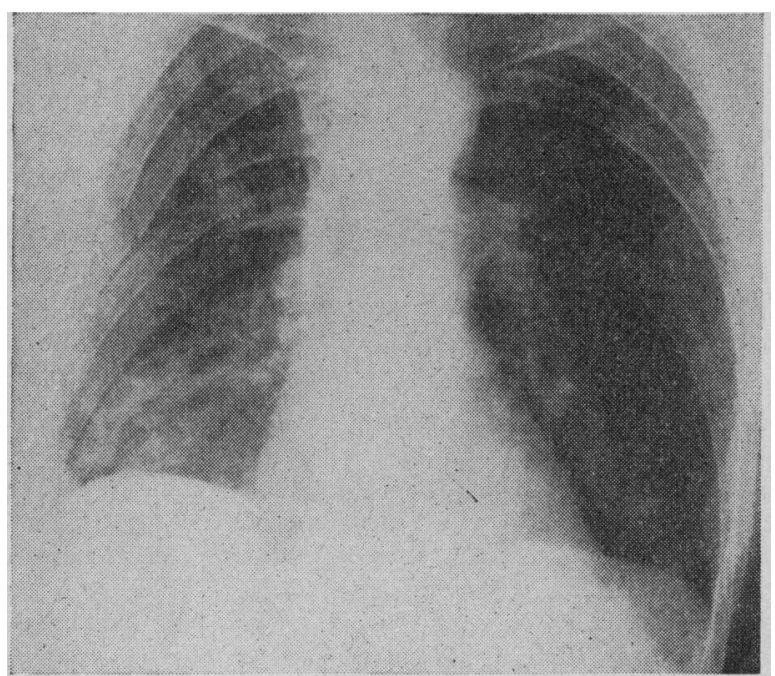

FIG. 1.-Case 4. Radiograph showing overriding of fractured ribs.

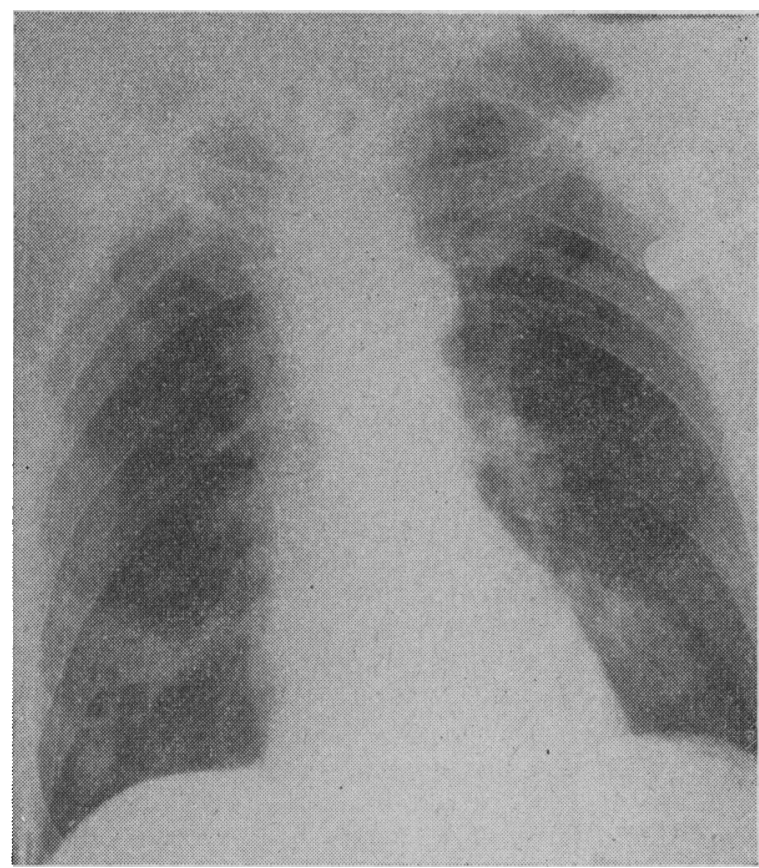

FIG. 2.-Case 4. Later radiograph showing correction of overriding. he continued to ride his bicycle without distress, his discharge $x$-ray film showed a considerable degree of traumatic thoracoplasty. The oxygen uptake on the affected side was $75 \%$ less than that on the unaffected one (Fig. 3).

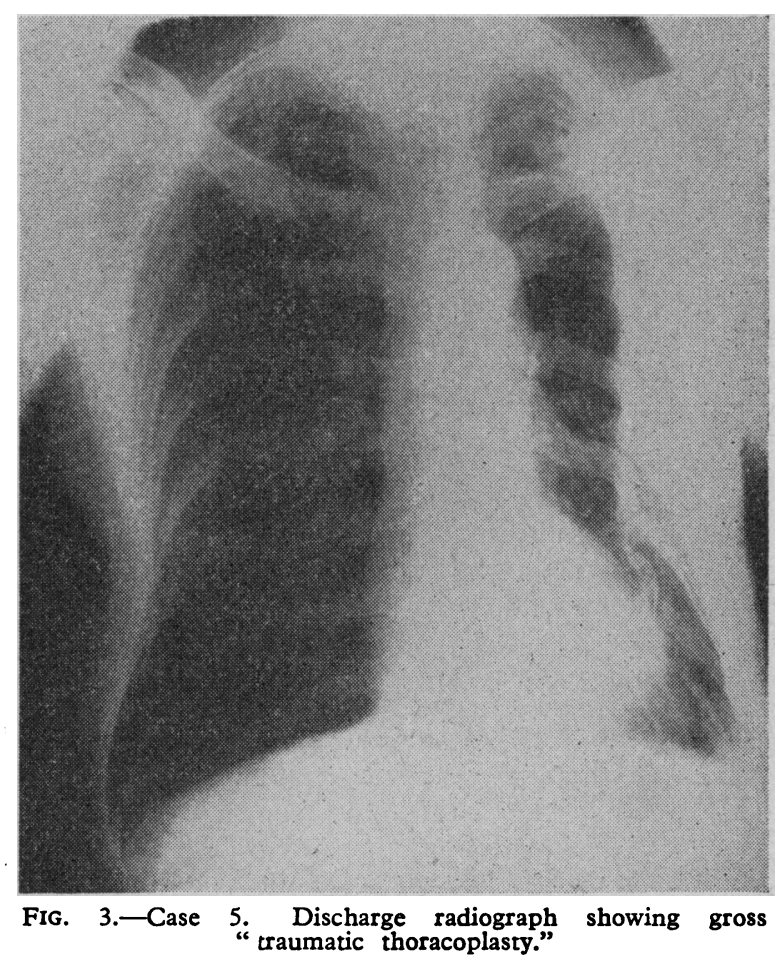

Case 6.-A 65-year-old man with a long history of chronic bronchitis and emphysema, who had been in right heart failure, was admitted with double fractures of the third to eighth ribs inclusive on the right side. Extradural analgesia was not considered because of the number of rib fractures, and he was unable to ventilate or

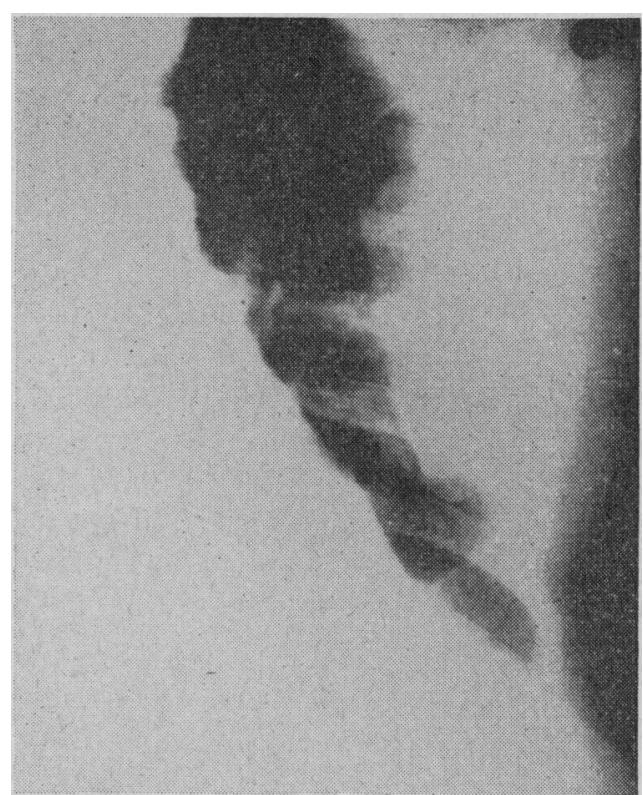

FIG. 4.-Case 6. Radiograph showing disimpaction of ribs at fracture sites.

clear his airways even after tracheotomy. I.P.P.R. was applied and 14 litres of oxygen-enriched air was necessary to prevent him from breathing out of phase with the respirator and to keep his $\mathrm{PCO}_{2}$ and $\mathrm{PO}_{2}$ within normal limits. Expiration occurred at only a high functional residual capacity, and disimpaction of the ribs at the fracture site was found to be present (Fig. 4). After I.P.P.R. was discontinued the ribs healed in a normal anatomical position. He developed a tracheal stenosis above the tracheostome, and it would have been impossible to close his tracheotomy without operative cure 
of the stenosis. As the patient maintained that his general condition was much better than before he had a tracheotomy, and as enough air could pass the stenosis to enable him to speak, no attempt has been made to remove the tracheotomy tube and he has been discharged.

\section{Discussion}

In Oxford the management of severe chest injuries is shared by the Accident Service and the Anaesthetic Department. Final clinical responsibility lies with the surgeon under whom the patient is admitted. If the condition of a patient with a chest injury gives cause for anxiety the casualty officer consults the duty anaesthetist. Senior registrar and consultant cover in both specialties is readily available.

We have found it helpful to group patients with chest injuries as described above, and we believe that by rationalizing our approach to each patient we have avoided unnecessarily complex forms of treatment. Extradural analgesia is proving increasingly valuable in the treatment of chest injuries, and it is our impression that more patients are avoiding tracheotomy because the complete relief from pain reduces paradoxical respiration and allows them to clear their lower airways by coughing. It is important, however, to be aware of the potential dangers of this method of treatment. Patients with large numbers of fractured ribs may be unsuitable for extradural analgesia because if pain is to be controlled the extent of the analgesia will cause hypotension by ganglionic blockade. This applies especially to hypovolaemic patients before resuscitation. It is probable that one of the deaths in our series was from severe hypotension following the injection of a small amount of local analgesic solution in a hypovolaemic patient with a large number of fractured ribs. In some circumstances it has been judged prudent to use conventional analgesics for 24 hours before beginning continuous extradural analgesia. On the other hand, it is our impression that in spontaneously breathing patients fractures of lower ribs are more painful than fractures of upper ribs, and a small injection of local analgesic aimed at the few painful fractures out of many has been very satisfactory.

The decision to place a patient in a particular group is based on signs which are clinical and biochemical, and which indicate whether respiratory function is adequate or not. The anatomical extent of a chest injury may be misleading in assessing functional severity. Some patients with only two or three fractured ribs cannot, in spite of pain relief and tracheotomy, clear their lower airways by coughing. Such inability has in our experience been due to age, pre-existing disease, lung contusion, or infection. In a few instances the impairment of normal ventilation resulting from retained secretions has made I.P.P.R. necessary to avoid respiratory failure.

The patient's respiratory rate and depth, the character of his respiration, his colour, and observation of his cough will give some indication of whether ventilation is adequate or not and whether it is likely to remain so. Observation of expired respiratory minute-volume and of the $\mathrm{PCO}_{2}, \mathrm{Po}_{2}$, and $p \mathrm{H}$ of arterial blood can be very helpful, but must be interpreted with care. For instance, in patients with chest injuries a low $\mathrm{PCO}_{2}$ does not necessarily mean that ventilation is adequate. We have seen patients with a $\mathrm{PcO}_{2}$ of $32-34 \mathrm{~mm}$. $\mathrm{Hg}$ whose $\mathrm{Po}_{2}$ was as low as $70 \mathrm{~mm}$. $\mathrm{Hg}$ and who were cyanosed. This is because in patients with lung contusion a large proportion of the cardiac output passes through the lungs exposed only to alveoli which are not being ventilated. Hyperventilation of the functionally intact parts of the lung can often remove enough $\mathrm{CO}_{2}$ to lower the $\mathrm{PCO}_{2}$ of arterial blood, but no amount of hyperventilation can raise arterial $\mathrm{PO}_{2}$ significantly. Presumably because there are as many poorly ventilated alveoli as completely unventilated ones, the addition of oxygen to the inspired air usually results in considerable improvement.

The East-Radcliffe respirator has been used to apply I.P.P.R. in the series of cases described, and has proved satisfactory.
Subatmospheric pressure during the expiratory phase of I.P.P.R. would defeat one of the objects of treatment, and it has never been used. We have been surprised that the increase in mean intrathoracic pressure during I.P.P.R. has not proved harmful even in hypovolaemic patients. It is presumed that these patients retain their circulatory reflexes, although we have no evidence to support this (Watson et al., 1962).

The East-Radcliffe respirator cannot be "triggered" by the patient, and, although we have no doubt that patienttriggered respirators can be successfully used in the treatment of chest injuries, we have not found the lack of this refinement a disadvantage. There is evidence that patients who are adequately ventilated will not breathe out of phase with a respirator (Spalding and Smith, 1963). It is important, therefore, to give the patient on I.P.P.R. enough respiratory minutevolume to keep $\mathrm{PCO}_{2}$ below $40 \mathrm{~mm} . \mathrm{Hg}$, and this may require a respiratory minute-volume of over $201 . / \mathrm{min}$. The patient's spontaneous respiratory minute-volume is often a guide to the ventilation which should be imposed. In Case 3 the patient's spontaneous minute-volume before I.P.P.R. was $18 \mathrm{l} . / \mathrm{min}$. at a frequency of $45 \mathrm{l} . / \mathrm{min}$. On I.P.P.R. he accepted the same minute-volume at a frequency of $20 \mathrm{l} . / \mathrm{min}$.- - less than half that which he had chosen when breathing spontaneously. Patients who have normal or low arterial $\mathrm{PCO}_{2}$ will breathe out of phase with the respirator if their $\mathrm{PO}_{2}$ falls below a certain level. We have watched patients who were being ventilated with oxygenenriched mixtures breathe out of phase with the respirator when the supplemental oxygen was removed and ventilation was with air alone. The lungs of one of our patients were so far destroyed by contusion and infection that it was impossible to reduce his $\mathrm{CO}_{2}$ below $40 \mathrm{~mm}$. $\mathrm{Hg}$ in spite of using the maximum output of the respirator. Patients like this will breathe out of phase with the respirator and by doing so will become even more difficult to ventilate. They are probably the only patients with chest injuries who should be paralysed with curare.

An indication for I.P.P.R. not dependent on the adequacy of ventilation is the onset of "traumatic thoracoplasty." In chest injuries early $x$-ray films may not disclose the full extent of the initial injury, which becomes apparent only when overriding of ribs occurs after two or three days. Cases 4 and 5 show the differences between similar patients treated with and without I.P.P.R. It is possible that the functional difference between these patients might be less than the radiographs suggested but lung-function studies performed on Case 5 showed oxygen uptake reduced by $75 \%$ on the affected side.

None of the patients in this series had any form of fixation of fractured ribs. Paradoxical respiration can be controlled and the anatomy of the rib cage restored by I.P.P.R., and it is possible that surgical fixation of ribs is no longer necessary. A fractured sternum, on the other hand, can be stabilized by an intramedullary nail. Thoracotomy, of course, may still be necessary to treat intrathoracic bleeding, ruptured bronchus, ruptured diaphragm, or intrapericardial bleeding.

\section{Summary}

The management of 121 cases of chest injury treated at the Radcliffe Infirmary in the past four years is described. The classification of patients into three groups of severity is recommended as an aid to the choice of appropriate treatment.

Some patients may be classified when first seen. In others the effect of emergency treatment of a number of conditions common to many chest injuries must first be observed. These conditions and their treatment are described.

Classification depends not on the anatomical extent of the injury but on the patient's ability to ventilate satisfactorily. This in turn depends on his ability to breathe and clear his airways by coughing. The efficiency of his cough must be judged clinically, but ventilation should be assessed by measurements of arterial $p \mathrm{H}, \mathrm{PCO}_{2}$, and $\mathrm{Po}_{2}$. 
Patients in group 1 are treated by pain relief alone. If this is not sufficient to maintain adequate ventilation, they require tracheotomy and fall into group 2. All those who still cannot ventilate adequately need I.P.P.R. and fall into group 3.

The maintenance of the treatment appropriate to each group is discussed, and it is emphasized that frequent assessments of ventilation should be made in order that deterioration shall not go unrecognized.

Pain can be completely controlled by thoracic segmental extradural block, and it is felt that the use of this technique decreases the need for tracheotomy or I.P.P.R., especially in the elderly and those with pre-existing chest disease.

Illustrative case reports are presented. The overall mortality in the series is $9.09 \%$.

We thank Mr. J. C. Scott, Director of the Accident Service, Radcliffe Infirmary, for his encouragement and for permission to publish details of patients; also the members of his staff who have co-operated in this form of treatment. We also thank Professor Sir Robert Macintosh and Dr. D. Maxwell for their advice in pre- paring this paper; Drs. R. Marshall and M. Rorke for their help in blood-gas estimations; and Dr. B. R. J. Simpson for the idea of continuous analgesia in chest injuries. We are grateful to Sister Howells and the nursing staff of the Accident Department for the very high standard of nursing care provided. The radiographs were prepared by $\mathrm{Mr}$. I. Roberts, of the Nuffield Department of Anaesthetics.

\section{REFERENCES}

Barrett, N. R. (1960). Lancet, 1, 293.

Campbell, E. J. M., and Howell, J. B. L. (1962). Brit. med. F., 2, 630. Denman, E. E., Cairns, C. S., and Holmes, C. McK. (1964). Ibid., 2, 101 .

Ferris, B. G., jun., and Pollard, D. S. (1960). F. clin. Invest., 39, 143.

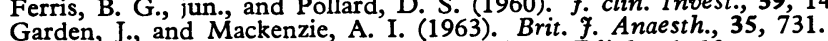

Garden, J., and Mackenzie, A. I. (1963). Brit. F. Anaesth.,

Griffiths, H. W. C. (1960). F. roy. Co

Nosworthy, M. D. (1951). Anaesthesia, 6, 211.

Simpson, B. R., Parkhouse, J., Marshall, R., and Lambrechts, W. (1961), Brit. F. Anaesth., 33, 628 .

Spalding, J. M. K. (1956). Lancet, 2, 1140

and Smith, A. C. (1963). Clinical Practice and Physiology of Artificial Respiration. Blackwell Oxford.

Watson, W. E., Smith, A. C., and Spalding, J. M. K. (1962). Brit. F. Anaesth 34, 278 . A

Windsor. H. M., and Dwyer, B. (1961). Thorax, 16, 3.

\title{
Cytological Diagnosis of Cancer : Its Uses and Limitations
}

\author{
M. M. BODDINGTON,* M.A., B.SC. ; A. I. SPRIGGS, † D.M., M.R.C.P.
}

Brit. med. F., 1965, 1, 1523-1529

The diagnosis of neoplastic disease still rests on the skilled interpretation of microscopical appearances. Although an empirical procedure, the examination of sections from a sufficiently large piece of tissue nearly always permits a trained observer to state correctly whether there is a malignant tumour or not. If tongues of neoplastic epithelium are seen growing down into the normal tissue of an organ, this may furnish a very convincing demonstration; but the histologist is often able to make a reliable diagnosis from a piece of solid tumour in which there is no normal tissue left to be invaded. He does this by an experienced judgment of the morphology of the cells and their arrangement relative to each other and to the stroma.

A diagnosis of malignancy made by examining smears of exudates, natural secretions, and scrapings is in general less reliable than one given by the histopathologist. But if a cytologist soberly bases his opinions on a sufficiently large past experience, and does not attempt inferences from appearances with which he is not familiar, he is able to detect malignancy in cases which otherwise would be missed, and sometimes to make a firm diagnosis in cases where the tumour is inaccessible to biopsy.

Now that the demand for cytological diagnosis of cancer is becoming more insistent many pathologists are put in the position of providing a service without feeling very sure of the value of it. In some centres cytodiagnosis has been used in one of its applications only, and the person responsible is in doubt whether to extend his service from, for example, sputum diagnosis to examine cervical smears or gastric washings as well. The literature on the whole consists of enthusiastic reports, and one does not always know whether to expect such good results in conditions of routine application.

This paper is intended to give a brief guide to what in our opinion are the most important applications of cytodiagnosis.

* Cytologist, Cyrodiagnostlc Unit, Churchill Hospital, Oxford.

† Consultant Cytologist, Cytodiagnostic Unit, Churchill Hospital, Oxford.
It is based partly on personal experience and partly on an examination of the literature.

The cytological method has two different fields of usefulness in clinical pathology. First, it can be used as a detection test for screening populations. When applied in this way it is only important that no case should slip through the net; accuracy of diagnosis is immaterial, as all suspicious cases will be fully investigated subsequently. Secondly, the cytological method may be used to give a definite diagnosis. In this case it is of vital importance that "positive" reports should be accurate; the observer is giving an opinion that a patient has malignant disease, and if the diagnosis later turns out wrong this is a serious mistake. When cytological reports are given it should be made quite clear whether the object is detection or diagnosis.

At Oxford the cytological method has been used for both these purposes. In the past 12 years 28,874 specimens have been examined for the detection of lesions of the female genital tract, and 22,609 specimens of various kinds for diagnostic purposes.

For the first five years all smears were scanned by one of us (mostly by M. M. B.), then one, two, and recently three technicians were employed. Since research programmes were undertaken concurrently, it is not possible to calculate from this the number of smears which can reasonably be examined by one person per day. McLaren (1963) suggests 30 per day as being within the limits set by fatigue, but in the early years of this series this was sometimes far exceeded. In the U.S.A. the number examined per technician per year is between 3,000 and 5,000 (Horn and Siegel, 1961).

\section{Method of Reporting}

We have used a flexible system of wording rather than the five classes of Papanicolaou. Where the object of the test is detection (cervical smears) there is no need for a categorical 